\title{
Finite-volume effects in moving frames
}

\section{Changhoan Kim*}

University of Southampton, School of Physics and Astronomy, Highfield, Southampton, SO17 1BJ, United Kingdom

E-mail: chateau@phys.soton.ac.uk

\section{Chris T. Sachrajda}

University of Southampton, School of Physics and Astronomy,

Highfield, Southampton, SO17 1BJ, United Kingdom

E-mail: cts@phys.soton.ac.uk

\section{Stephen R. Sharpe}

University of Washington, Department of Physics, Seattle, WA-98195-1560, USA

E-mail: sharpeephys.washington.edu

\begin{abstract}
We determine the quantization condition for the energy levels of two interacting particles in a finite box in a "moving frame", i.e. one in which the total momentum of pions is non-zero. This condition is valid up to corrections which fall exponentially withe the box size, and holds only below the inelastic threshold. It is derived using field theoretic methods, using a generalization of previous summation formulae relating sums and integrals over momenta. The result agrees with that obtained earlier by Rummakainen and Gottlieb using a relativistic quantum mechanical approach. Technically, we expand the finite-volume four-point Green function in terms of the infinite-volume Bethe-Salpeter kernel, and determine the position of the poles. The final result is written in terms of the two-pion scattering phase shift. Our result can be used to facilitate the determination of the scattering phase shift, and can be used to generalize the Lellouch-Lüscher formula relating finite-volume two-particle matrix elements to those in infinite volume.
\end{abstract}

XXIIIrd International Symposium on Lattice Field Theory

25-30 July 2005

Trinity College, Dublin, Ireland

\footnotetext{
${ }^{*}$ Speaker.
} 


\section{Introduction}

Lattice simulations are necessarily performed in a finite volume. Unlike the exponentially small errors in lattice evaluation of hadronic masses or matrix elements with at most a single hadron in the external states, the errors when two (or more) hadrons are present in external states the finitevolume effects decrease more slowly, as powers of the box size, $L$, and need to be understood in order to obtain physical quantities with good precision. The theory of such effects has been fully developed for two particles in their rest frame, i.e. with total momentum $\vec{P}=0$. The spectrum of such states was worked out in refs. [2, 3, 4] and the finite volume corrections to the matrix elements were obtained in refs. [5, 6].

In this note, we report on a determination of the spectrum of two-particle states with total momentum $\vec{P} \neq 0$, which we call a moving frame. ${ }^{1}$ Full details are given in ref. [8]. ${ }^{2}$

There are several reasons why this extension is important, e.g. the use of a moving frame obviates the need for vacuum subtractions in the isoscalar channel, which is otherwise under poor statistical control. Further applications are discussed in refs. [7, 8].

The energy levels of two-particle system are determined from the position of poles of corresponding correlation function. By expanding the correlation function in terms of Bethe-Salpeter kernel, we can locate the terms which potentially generate power corrections. With the aid of summation formulae, we can calculate these corrections up to exponentially small errors. After rearranging and resumming we deduce the quantization condition, whose solutions generate the location of poles. This condition depends on the scattering phase shift and the box size. Thus, we can determine the scattering phase shift from a lattice calculation of spectrum. We sketch the derivation of the summation formulae in sec. 2 and of the quantization condition in sec. 3 .

\section{Summation Formulae}

Finite-volume effects for two-hadron states originate from the difference between the sums over the discrete momenta in a finite volume and the corresponding integrals over the continuous spectrum in infinite volume. To determine the relation between them, we start from the Poisson summation formula,

$$
\frac{1}{L^{3}} \sum_{\vec{k}} g(\vec{k})=\sum_{\vec{l}} \int \frac{d^{3} k}{(2 \pi)^{3}} e^{i L \vec{l} \cdot \vec{k}} g(\vec{k}) \approx \int \frac{d^{3} k}{(2 \pi)^{3}} g(\vec{k})+O\left(e^{-L}\right)
$$

where the summation on the left-hand-side is over all integer values of $\vec{n}=\left(n_{1}, n_{2}, n_{3}\right)$, with $\vec{k}=$ $(2 \pi / L) \vec{n}$. The approximation is valid when the Fourier transform of $g(\vec{k}),, \tilde{g}(\vec{r})$, is non-singular, and is either contained in a finite spatial region or decreases exponentially as $|\vec{r}| \rightarrow \infty$. We note that functions $g(\vec{k})$ with these properties have no singularities for real $\vec{k}$, and fall off fast enough at $|\vec{k}| \rightarrow \infty$ that the integrals in eq. (2.1) converge.

\footnotetext{
${ }^{1} \mathrm{~A}$ generalisation of the results for the spectrum of such states to a moving frame was proposed some time ago in ref. [7]. We address this in sec. 4

${ }^{2}$ The results have been also obtained in ref. [9] using a different method.
} 
However, the finite-volume corrections for two-hadron correlators with non-zero total momentum are contained in summations of the form

$$
S\left(q^{*}\right) \equiv \frac{1}{L^{3}} \sum_{\vec{k}} \frac{\omega_{k}^{*}}{\omega_{k}} \frac{f\left(\vec{k}^{*}\right)}{q^{* 2}-k^{* 2}} .
$$

where we assume that $q^{* 2}$ is such that there is no term in the sum with $k^{* 2} \equiv\left|\overrightarrow{k^{*}}\right|^{2}=q^{* 2}$ and that $f\left(\overrightarrow{k^{*}}\right)$ has the properties discussed above. Note that the summation is over the moving frame momenta $\vec{k}=(2 \pi / L) \vec{n}$, with $\vec{n}$ being a vector of integers but the summand is written in terms of the centre-of-mass momenta $\vec{k}^{*}$ using the Lorentz transformation of eq.(3.4). It is also convenient, as will be apparent shortly, to pull the Jacobian $\omega_{k}^{*} / \omega_{k}$ out of the function $f\left(\vec{k}^{*}\right)$.

The manifest singularity at $k^{2}=q^{2}$ forbids the immediate application of the eq. (2.1). In order to avoid this difficulty, we expand $f$ in terms of spherical harmonics ${ }^{3}$ and subtract from $S\left(q^{*}\right)$ a function which is specially chosen to cancel the singularity:

$$
\begin{aligned}
& \frac{1}{L^{3}} \sum_{\vec{k}} J \frac{f_{l m}\left(k^{*}\right)-f_{l m}\left(q^{*}\right) e^{\alpha\left(q^{* 2}-k^{* 2}\right)}}{q^{* 2}-k^{* 2}} k^{* l} \sqrt{4 \pi} Y_{l m}\left(\theta^{*}, \phi^{*}\right) \\
& =\int \frac{d^{3} k}{(2 \pi)^{3}} J \frac{f_{l m}\left(k^{*}\right)-f_{l m}\left(q^{*}\right) e^{\alpha\left(q^{* 2}-k^{* 2}\right)}}{q^{* 2}-k^{* 2}} k^{* l} \sqrt{4 \pi} Y_{l m}\left(\theta^{*}, \phi^{*}\right) \\
& =\int \frac{d^{3} k^{*}}{(2 \pi)^{3}} \frac{f_{l m}\left(k^{*}\right)-f_{l m}\left(q^{*}\right) e^{\alpha\left(q^{* 2}-k^{* 2}\right)}}{q^{* 2}-k^{* 2}} k^{* l} \sqrt{4 \pi} Y_{l m}\left(\theta^{*}, \phi^{*}\right) .
\end{aligned}
$$

where the Jacobian factor $\omega_{k}^{*} / \omega_{k}$ corresponds to the change of integration variables from the laboratory-frame momenta $\vec{k}$ to the centre-of-mass frame momenta $\vec{k}^{*}$. This relation is valid up to terms which are exponentially small in the volume. The exponential factors $\exp \left[\alpha\left(q^{* 2}-k^{* 2}\right)\right]$ (with $\alpha>0$ ) are included so that the subtraction does not introduce ultraviolet divergences. By rearranging terms, the required summation formulae for given spherical harmonics is 4

$$
S_{l m}\left(q^{*}\right)=\delta_{l, 0} \mathscr{P} \int \frac{d^{3} k^{*}}{(2 \pi)^{3}} \frac{f_{00}\left(k^{*}\right)}{q^{* 2}-k^{* 2}}+f_{l m}\left(q^{*}\right) c_{l m}^{P}\left(q^{* 2}\right)
$$

where

$$
c_{l m}^{P}\left(q^{* 2}\right)=\frac{1}{L^{3}} \sum_{\vec{k}} \frac{e^{\alpha\left(q^{* 2}-k^{* 2}\right)}}{q^{* 2}-k^{* 2}} k^{* l} \sqrt{4 \pi} Y_{l m}\left(\theta^{*}, \phi^{*}\right)-\delta_{l, 0} \mathscr{P} \int \frac{d^{3} k^{*}}{(2 \pi)^{3}} \frac{e^{\alpha\left(q^{* 2}-k^{* 2}\right)}}{q^{* 2}-k^{* 2}} .
$$

Although the integrand in eq. (2.4) has no pole at $k=q$, in eq. (2.5) we separate it into two terms each of which does have such a pole. For consistency, the two terms need to be regulated in the same way and the principal value prescription, denoted $\mathscr{P}$, is a natural choice. Finally, we gather all the spherical harmonics and then the summation formula is

$$
S\left(q^{*}\right)=\mathscr{P} \int \frac{d^{3} k^{*}}{(2 \pi)^{3}} \frac{f\left(\vec{k}^{*}\right)}{q^{* 2}-k^{* 2}}+\sum_{l=0}^{\infty} \sum_{m=-l}^{l} f_{l m}\left(q^{*}\right) c_{l m}^{P}\left(q^{* 2}\right) .
$$

\footnotetext{
${ }^{3}$ Here, we used $\sqrt{4 \pi} Y_{l m}$, which simplifies the normalization for $l=0$.

${ }^{4}$ This equality holds up to exponentially small corrections. From now on, this will not be stated explicitly.
} 


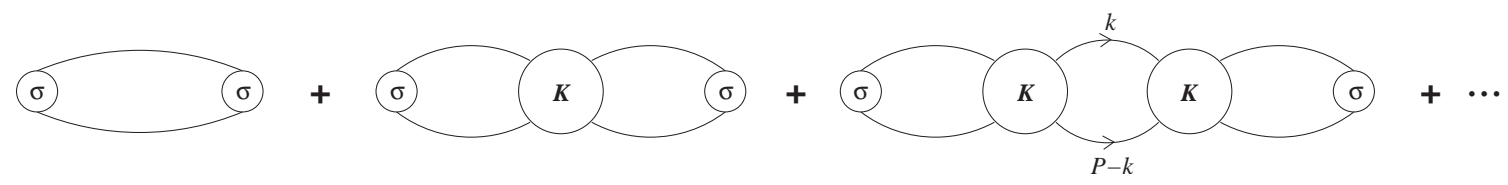

Figure 1: Diagrammatic expansion of the correlator $\widetilde{C}_{\vec{P}}(E)$. Propagators are fully dressed and normalized to unity on shell. $K$ is the amputated two-particle irreducible four-particle correlation function. The circles at the ends represent the operator $\sigma$, renormalized by two factors of $\sqrt{Z}$.

\section{Quantization Condition in Moving Frames}

The two-pion spectrum in finite volume can be extracted from the correlation functions of composite operators:

$$
C_{\vec{P}}(t)=\left\langle 0\left|\sigma_{\vec{P}}(t) \sigma^{\dagger}(\overrightarrow{0}, 0)\right| 0\right\rangle, \quad \widetilde{C}_{\vec{P}}(E)=\int d t e^{-i E t} C_{\vec{P}}(t) .
$$

(with time ordering implicit), where $\sigma(\vec{x}, t)$ is an interpolating operator for two-pion states and $\sigma_{\vec{P}}(t)$ is its spatial Fourier transform. We will determine the quantization condition by identifying the position of poles of $\widetilde{C}_{\vec{P}}(E)$.

The correlation function $\widetilde{C}_{\vec{P}}$ can be expressed in terms of the Bethe-Salpeter kernel $K$ through the series shown in fig.1. Since we choose $E$ to lie below the four-pion threshold, there are no intermediate states with four or more pions and the finite-volume effects in $K$ are exponentially suppressed [2, 6]. The same is true of the dressed single particle propagators [1]. The only powerlaw volume corrections arise through the the two pion loops, and we now turn to an analysis of these corrections. The generic loop integration/summation appearing in fig. 1 is of the form ${ }^{5}$

$$
I \equiv \frac{1}{L^{3}} \sum_{\vec{k}} \int \frac{d k_{0}}{2 \pi} \frac{f\left(k_{0}, \vec{k}\right)}{\left(k^{2}-m^{2}+i \varepsilon\right)\left((P-k)^{2}-m^{2}+i \varepsilon\right)}
$$

where $k=\left(k_{0}, \vec{k}\right)$ and $P=(E, \vec{P})$ are four vectors and we have left out a factor of $i^{2}=-1$ from the propagators which will be accounted for later. The only properties of $f$ that we need are, first, that it has no singularities for real $\vec{k}$ (which holds given our kinematical constraint on $E$ ), and, second, that its ultraviolet behaviour is such as to render the integral and sum convergent.

To simplify the pole structure we first perform the $k_{0}$ integration. We choose to close the contour of integration so as to pick up the "particle" contribution from the first pole and the "antiparticle" contribution from the second:

$$
I=-i \frac{1}{L^{3}} \sum_{\vec{k}}\left\{\frac{f\left(\omega_{k}, \vec{k}\right)}{2 \omega_{k}\left(\left(E-\omega_{k}\right)^{2}-\omega_{P k}^{2}\right)}+\frac{f\left(E+\omega_{P k}, \vec{k}\right)}{2 \omega_{P k}\left(\left(E+\omega_{P k}\right)^{2}-\omega_{k}^{2}\right)}\right\},
$$

where $\omega_{k}=\sqrt{\vec{k}^{2}+m^{2}}$ and $\omega_{P k}=\sqrt{(\vec{P}-\vec{k})^{2}+m^{2}}$.

\footnotetext{
${ }^{5}$ Although we phrase our discussion in Minkowski space, we note that this same object may be obtained from the Euclidean space correlators calculated in lattice simulations by analytic continuation to imaginary Euclidean energy (which is the approach used in ref. [2]).
} 
For the kinematic region of interest, $0<E^{2}-P^{2}<16 m^{2}$, it is straightforward to show that the only singularity in $I$ is the explicit pole in the first term inside the braces in eq. (3.3), which occurs at those values of $E$ for which there is a term in the summation with $\omega_{k}+\omega_{P k}=E$. Since this singularity leads to finite-volume corrections which decrease like powers of the volume, we need to examine the first term in more detail.

With Lorentz transformed variables,

$$
k_{\|}^{*}=\gamma\left(k_{\|}-\beta \omega_{k}\right), \quad \tilde{k}_{\perp}^{*}=\tilde{k}_{\perp} \quad \text { and } \quad \omega_{k}^{*}=\sqrt{k^{* 2}+m^{2}}=\gamma\left(\omega_{k}-\beta k_{\|}\right) .
$$

we rewrite the first term (which we call $I_{1}$ ) in the form

$$
I_{1}=-i \frac{1}{L^{3}} \frac{1}{E^{*}} \sum_{\vec{k}} \frac{1}{2 \omega_{k}} \frac{f^{*}\left(\vec{k}^{*}\right)}{E^{*}-2 \omega_{k}^{*}}=-i \frac{1}{L^{3}} \frac{1}{2 E^{*}} \sum_{\vec{k}} \frac{\omega_{k}^{*}}{\omega_{k}} \frac{f^{*}\left(\vec{k}^{*}\right)}{q^{* 2}-k^{* 2}} \frac{E^{*}+2 \omega_{k}^{*}}{4 \omega_{k}^{*}},
$$

where $f^{*}$ is the function $f$ rewritten in terms of the centre-of-mass variables. We now apply the summation formulae from sec. 2 ,

$$
I_{1}=-i \frac{1}{2 E^{*}} \mathscr{P} \int \frac{d^{3} k^{*}}{(2 \pi)^{3}} \frac{f^{*}\left(\vec{k}^{*}\right)}{q^{* 2}-k^{* 2}} \frac{E^{*}+2 \omega_{k}^{*}}{4 \omega_{k}^{*}}-\frac{i}{2 E^{*}} \sum_{l=0}^{\infty} \sum_{m=-l}^{l} f_{l m}^{*}\left(q^{*}\right) c_{l m}^{P}\left(q^{* 2}\right) .
$$

In order to isolate the finite-volume correction, we replace the principal-value integral in eq. (3.6) by the corresponding integral with the Feynman ic prescription in the propagator and a "delta-function" term:

$$
I_{1}=-i \frac{1}{2 E^{*}} \int \frac{d^{3} k^{*}}{(2 \pi)^{3}} \frac{f^{*}\left(\vec{k}^{*}\right)}{q^{* 2}-k^{* 2}+i \varepsilon} \frac{E^{*}+2 \omega_{k}^{*}}{4 \omega_{k}^{*}}+\frac{q^{*} f_{00}^{*}\left(q^{*}\right)}{8 \pi E^{*}}-\frac{i}{2 E^{*}} \sum_{l=0}^{\infty} \sum_{m=-l}^{l} f_{l m}^{*}\left(q^{*}\right) c_{l m}^{P}\left(q^{* 2}\right) .
$$

Note that the "delta-function" term picks out the $l=0$ part of $f^{*}$. Observing that the first term in eq. (3.7) is exactly the infinite volume expression for $I_{1}$ in Minkowski space (after retracing the steps in the derivation above), we arrive at the finite-volume correction of loop integrals,

$$
I=I_{\infty}+I_{F V}, \quad I_{F V}=\left\{\frac{q^{*} f_{00}^{*}\left(q^{*}\right)}{8 \pi E^{*}}-\frac{i}{2 E^{*}} \sum_{l=0}^{\infty} \sum_{m=-l}^{l} f_{l m}^{*}\left(q^{*}\right) c_{l m}^{P}\left(q^{* 2}\right)\right\} .
$$

Since each loop integral (sum) in fig. 1 contains an infinite volume part and the finite volume correction, we can rearrange the expansion according to the number of insertions of the latter. Ignoring the zero insertion term, which is irrelevant to the pole structure of the correlation function, we can represent the series as in fig. 2, leading to the general result:

$$
\widetilde{C}_{\vec{P}}^{F V}(E)=-A^{\prime} F A+A^{\prime} F(i M / 2) F A+\cdots=-A^{\prime} F \frac{1}{1+i M F / 2} A .
$$

Here we have taken into account the factor of $i^{2}$ dropped from the loop in the previous section, as well as symmetry factors of $1 / 2$ arising from the identical nature of the particles. $A$ and $A^{\prime}$ represent the overlap of $\sigma$ with asymptotic two-pion states with definite angular momentum. $F$ is a kinematic factor obtained from $I_{F V}$ :

$$
F_{l_{1}, m_{1} ; l_{2}, m_{2}}=\frac{q^{*}}{8 \pi E^{*}}\left(\delta_{l_{1} l_{2}} \delta_{m_{1} m_{2}}-i \frac{4 \pi}{q^{*}} \sum_{l=0}^{\infty} \sum_{m=-l}^{l} \frac{\sqrt{4 \pi}}{q^{* l}} c_{l m}^{P}\left(q^{* 2}\right) \int d \Omega^{*} Y_{l_{1}, m_{1}}^{*} Y_{l, m}^{*} Y_{l_{2}, m_{2}}\right),
$$




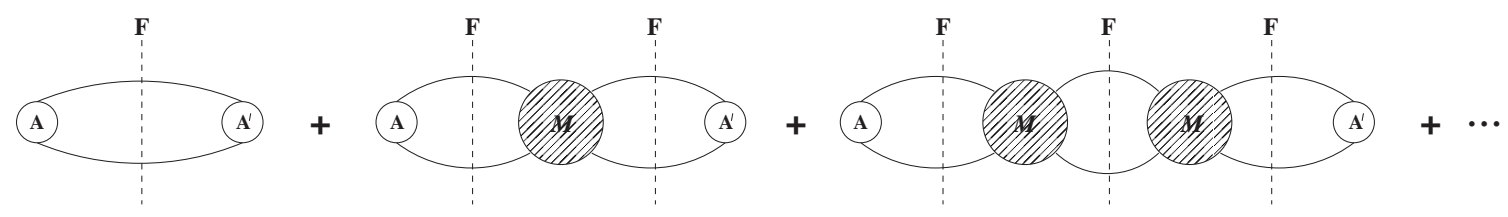

Figure 2: Contributions to the volume dependent part of the $\sigma$ correlator, $\widetilde{C}_{\vec{P}}^{F V}$. The notation is as in fig. 1 except that the filled circles represent the full scattering amplitude, $M$, given by a geometric sum of any number of insertions of the kernel $K$, and the vertical dashed lines indicate that the on-shell finite volume part, $I_{F V}$, has been used for the loop integral. The quantities $A$ and $A^{\prime}$ are defined in the text.

and $M$ is the on-shell scattering amplitude:

$$
M_{l_{1}, m_{1} ; l_{2}, m_{2}}=\delta_{l_{1} l_{2}} \delta_{m_{1} m_{2}} \frac{16 \pi E^{*}}{q^{*}} \frac{\left(\exp \left[2 i \delta_{l_{1}}\left(q^{*}\right)\right]-1\right)}{2 i},
$$

Now, we can determine the final quantization condition:

$$
\operatorname{det}(1+i M F / 2)=0 .
$$

Solving this after truncation of the partial wave expansion leads to results which can be shown to be equivalent to those of ref. [7].

\section{Conclusion}

We have provided a field theoretic derivation of the finite volume energy shift for two hadron states in a moving frame, confirming the result obtained by ref. [7]. Our result can be used to determine the finite-volume corrections to matrix elements of local composite operators with an initial and/or final state consisting of two hadrons, thus generalizes the Lellouch-Lüscher factor to moving frames [8, 9]. The path is now open to numerical studies of two pion energies and matrix elements in a moving frame, which, as discussed in refs. [7, 8, 9], simplifies a number of important applications.

\section{References}

[1] M. Luscher, Commun. Math. Phys. 104 (1986) 177.

[2] M. Luscher, Commun. Math. Phys. 105 (1986) 153.

[3] M. Luscher, Nucl. Phys. B 354 (1991) 531.

[4] M. Luscher, Nucl. Phys. B 364 (1991) 237.

[5] L. Lellouch and M. Luscher, Commun. Math. Phys. 219, 31 (2001) [arXiv:hep-lat/0003023].

[6] C. J. D. Lin, G. Martinelli, C. T. Sachrajda and M. Testa, Nucl. Phys. B 619 (2001) 467 [arXiv:hep-lat/0104006].

[7] K. Rummukainen and S. A. Gottlieb, Nucl. Phys. B 450 (1995) 397 [arXiv:hep-lat/9503028].

[8] C. h. Kim, C. T. Sachrajda and S. R. Sharpe [arXiv:hep-lat/0507006].

[9] N. H. Christ, C. Kim and T. Yamazaki, arXiv:hep-lat/0507009. 